\title{
Heterometallic group 4-lanthanide oxo-alkoxide precursors for synthesis of binary oxide nanomaterials
}

\author{
Rafał Petrus, ${ }^{\text {a }}$ Katarzyna Chomiak, ${ }^{\mathrm{b}}$ Józef Utko, ${ }^{\mathrm{c}}$ Alina Bieńko, ${ }^{\mathrm{c}}$ Tadeusz Lis, ${ }^{\mathrm{c}}$ and Piotr \\ Sobota ${ }^{* b}$
}

${ }^{\text {aF }}$ aculty of Chemistry, Wrocław University of Science and Technology, 23 Smoluchowskiego, 50-370 Wrocław, Poland

${ }^{\text {b } Ł u k a s i e w i c z ~ R e s e a r c h ~ N e t w o r k ~-~ P O R T ~ P o l i s h ~ C e n t e r ~ F o r ~ T e c h n o l o g y ~ D e v e l o p m e n t, ~} 147$ Stablowicka, 54-066 Wrocław, Poland

${ }^{\mathrm{c}}$ Faculty of Chemistry, University of Wrocław, 14 F. Joliot-Curie, 50-383 Wrocław, Poland

\section{Corresponding author:}

Dr. Rafał Petrus, rafal.petrus@pwr.edu.pl

Prof. Dr. Piotr Sobota, piotr.sobota@port.org.pl

\section{Contents}

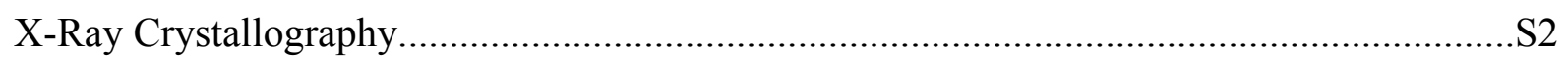

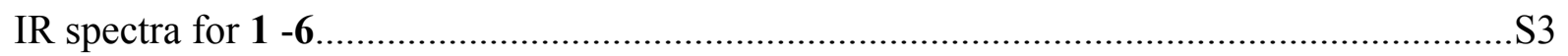

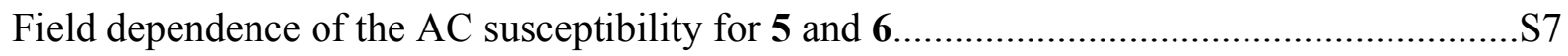


Crystallographic Data for Compounds 1-7.

Table S1. Crystal and data collection parameters for compounds 1-3.

\begin{tabular}{|c|c|c|c|}
\hline$\overline{\text { Crystal }}$ & $\mathbf{1} \cdot 2 \mathrm{EtOH}$ & 2 & 3 \\
\hline Chemical formula & $\mathrm{C}_{36} \mathrm{H}_{92} \mathrm{Cl}_{2} \mathrm{La}_{2} \mathrm{O}_{20} \mathrm{Ti}_{4}$ & $\mathrm{C}_{22} \mathrm{H}_{59} \mathrm{Cl}_{5} \mathrm{La}_{2} \mathrm{O}_{12} \mathrm{Zr}_{2}$ & $\mathrm{C}_{22} \mathrm{H}_{59} \mathrm{Cl}_{5} \mathrm{La}_{2} \mathrm{O}_{12} \mathrm{Hf}_{2}$ \\
\hline Formula Mass & 1477.55 & 1153.20 & 1327.74 \\
\hline Crystal system & Monoclinic & Monoclinic & Monoclinic \\
\hline Space group & $P 2_{1} / \mathrm{n}$ & $P 2_{1} / \mathrm{c}$ & $P 2_{1} / \mathrm{c}$ \\
\hline$a / \AA$ & $13.723(2)$ & $11.449(4)$ & $11.433(3)$ \\
\hline$b / \AA$ & $11.707(2)$ & $21.269(6)$ & $21.241(10)$ \\
\hline$c / \AA$ & $20.252(3)$ & $17.968(5)$ & $18.006(5)$ \\
\hline \multicolumn{4}{|l|}{$\alpha /^{\circ}$} \\
\hline$\beta /{ }^{\circ}$ & $99.21(2)$ & 107.64(3) & $107.70(3)$ \\
\hline \multicolumn{4}{|l|}{$\gamma /{ }^{\circ}$} \\
\hline Unit cell volume $/ \AA^{3}$ & $3211.6(9)$ & $4170(2)$ & $4166(3)$ \\
\hline Temperature/K & $100(2)$ & $100(2)$ & $100(2)$ \\
\hline$Z$ & 2 & 4 & 4 \\
\hline Radiation type & $\mathrm{MoK} \alpha$ & $\mathrm{MoK} \alpha$ & $\mathrm{MoK} \alpha$ \\
\hline Absorption coefficient, $\mu / \mathrm{mm}^{-1}$ & 1.92 & 2.86 & 7.343 \\
\hline No. of reflections measured & 18791 & 26992 & 18047 \\
\hline No. of independent reflections & 8955 & 9748 & 7734 \\
\hline No. of observed reflections $(I>2 \sigma(I))$ & 7617 & 6723 & 5519 \\
\hline$R_{\text {int }}$ & 0.0266 & 0.0905 & 0.0611 \\
\hline Final $R_{l}$ values $(I>2 \sigma(I))$ & 0.0308 & 0.0702 & 0.0587 \\
\hline Final $w R\left(F^{2}\right)$ values $(I>2 \sigma(I))$ & 0.0717 & 0.1556 & 0.1131 \\
\hline Final $R_{I}$ values (all data) & 0.042 & 0.1092 & 0.0931 \\
\hline Final $w R\left(F^{2}\right)$ values (all data) & 0.0769 & 0.1774 & 0.1297 \\
\hline Goodness of fit on $F^{2}$ & 1.046 & 1.040 & 1.051 \\
\hline$\Delta \rho \max / \mathrm{e} \AA^{-3}$ & 1.60 & 1.57 & 1.87 \\
\hline$\Delta \rho \min / \AA^{-3}$ & -0.87 & -2.15 & -2.21 \\
\hline
\end{tabular}


Table S2. Crystal and data collection parameters for compounds 5-7.

\begin{tabular}{|c|c|c|c|}
\hline$\overline{\text { Crystal }}$ & 5 & 6 & $7 \cdot 0.5 \mathrm{THF}$ \\
\hline Chemical formula & $\mathrm{C}_{56} \mathrm{H}_{150} \mathrm{Cl}_{6} \mathrm{Nd}_{4} \mathrm{O}_{30} \mathrm{Zr}_{4}$ & $\mathrm{C}_{56} \mathrm{H}_{150} \mathrm{Cl}_{6} \mathrm{Nd}_{4} \mathrm{O}_{30} \mathrm{Hf}_{4}$ & $\mathrm{C}_{58} \mathrm{H}_{152} \mathrm{Cl}_{3} \mathrm{Nd}_{5} \mathrm{O}_{32.5} \mathrm{Zr}_{4}$ \\
\hline Formula Mass & 2458.29 & 2807.37 & 2562.22 \\
\hline Crystal system & Triclinic & Triclinic & Monoclinic \\
\hline Space group & $P-1$ & $P-1$ & $P 2_{1} / \mathrm{c}$ \\
\hline$a / \AA$ & $11.214(3)$ & $11.372(4)$ & $14.660(2)$ \\
\hline$b / \AA$ & $12.755(3)$ & $13.145(5)$ & $19.998(3)$ \\
\hline$c / \AA$ & $18.994(4)$ & $18.847(5)$ & $33.693(6)$ \\
\hline$\alpha /^{\circ}$ & $72.36(3)$ & $72.74(3)$ & \\
\hline$\beta /^{\circ}$ & $78.20(2)$ & $77.08(2)$ & $94.24(2)$ \\
\hline$\gamma /{ }^{\circ}$ & $69.72(2)$ & $68.17(3)$ & \\
\hline Unit cell volume $/ \AA^{3}$ & $2413.5(11)$ & 2477.1(16) & $9851(3)$ \\
\hline Temperature/K & $100(2)$ & $190(2)$ & $150(2)$ \\
\hline$Z$ & 1 & 1 & 4 \\
\hline Radiation type & $\mathrm{MoK} \alpha$ & $\mathrm{MoK} \alpha$ & $\mathrm{MoK} \alpha$ \\
\hline Absorption coefficient, $\mu / \mathrm{mm}^{-1}$ & 2.75 & 6.45 & 3.14 \\
\hline No. of reflections measured & 22888 & 17099 & 60233 \\
\hline No. of independent reflections & 9967 & 10779 & 21059 \\
\hline No. of observed reflections $(I>2 \sigma(I))$ & 8789 & 8441 & 14271 \\
\hline$R_{\text {int }}$ & 0.0446 & 0.0283 & 0.0642 \\
\hline Final $R_{l}$ values $(I>2 \sigma(I))$ & 0.0528 & 0.0367 & 0.0569 \\
\hline Final $w R\left(F^{2}\right)$ values $(I>2 \sigma(I))$ & 0.1332 & 0.0682 & 0.1310 \\
\hline Final $R_{I}$ values (all data) & 0.0599 & 0.0535 & 0.0937 \\
\hline Final $w R\left(F^{2}\right)$ values (all data) & 0.1399 & 0.0765 & 0.1598 \\
\hline Goodness of fit on $F^{2}$ & 1.077 & 1.023 & 1.057 \\
\hline$\Delta \rho \max / \mathrm{e}^{-3}$ & 2.34 & 1.16 & 1.52 \\
\hline$\Delta \rho \min / \mathrm{e}^{-3}$ & -2.02 & -0.98 & -1.29 \\
\hline
\end{tabular}




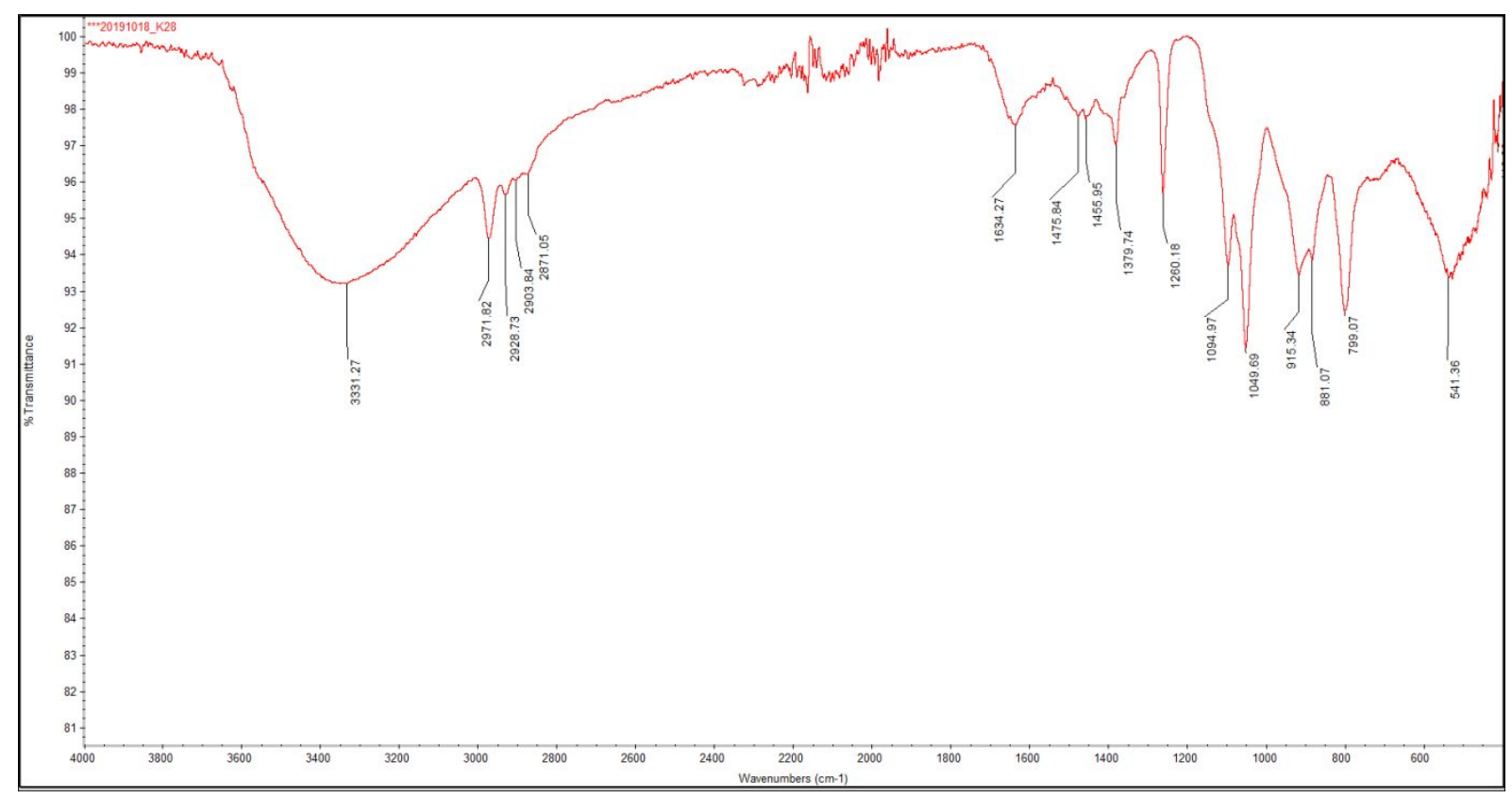

Figure S1. FTIR-ATR spectra of 1 .

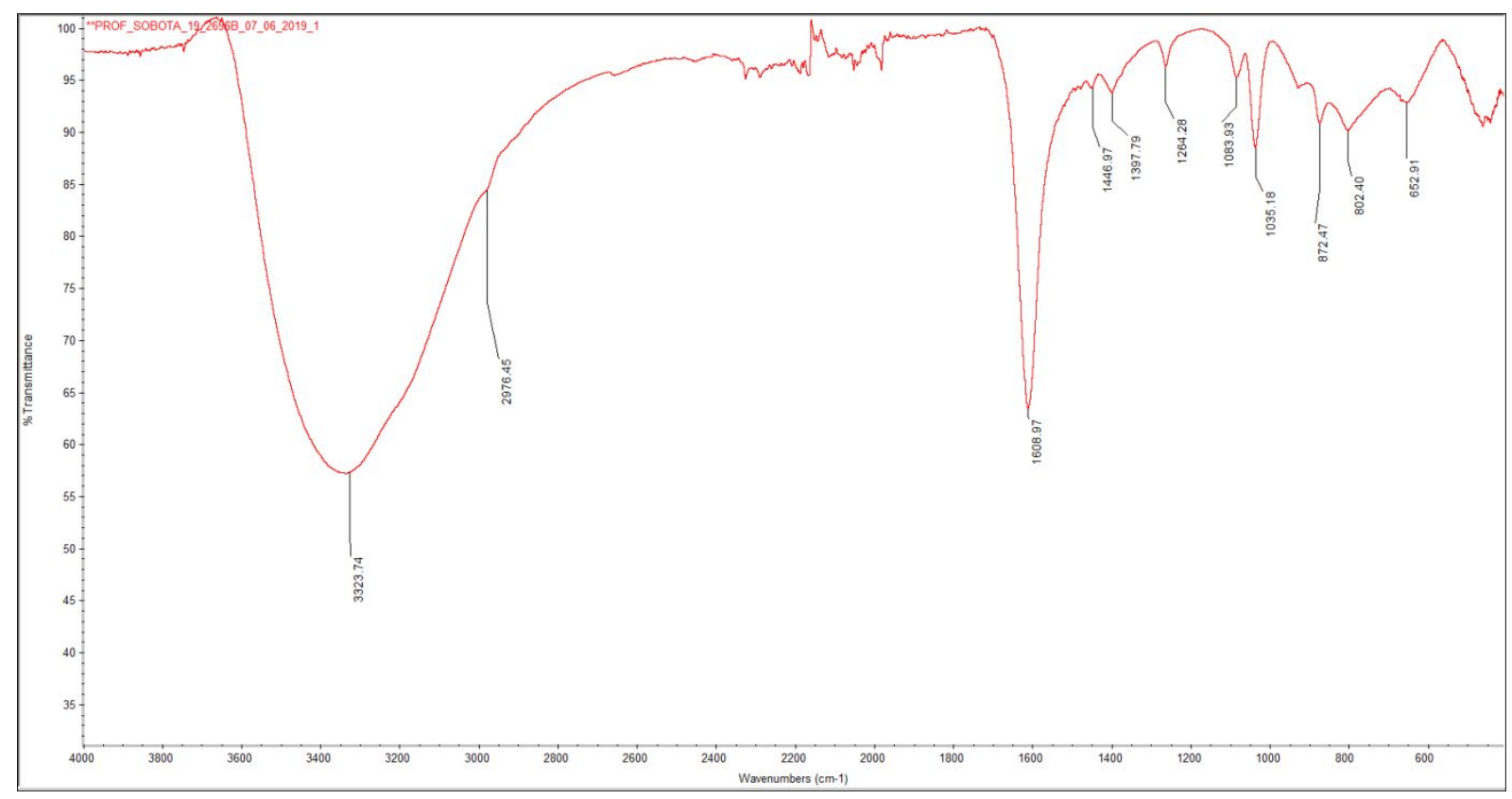

Figure S2. FTIR-ATR spectra of 2. 


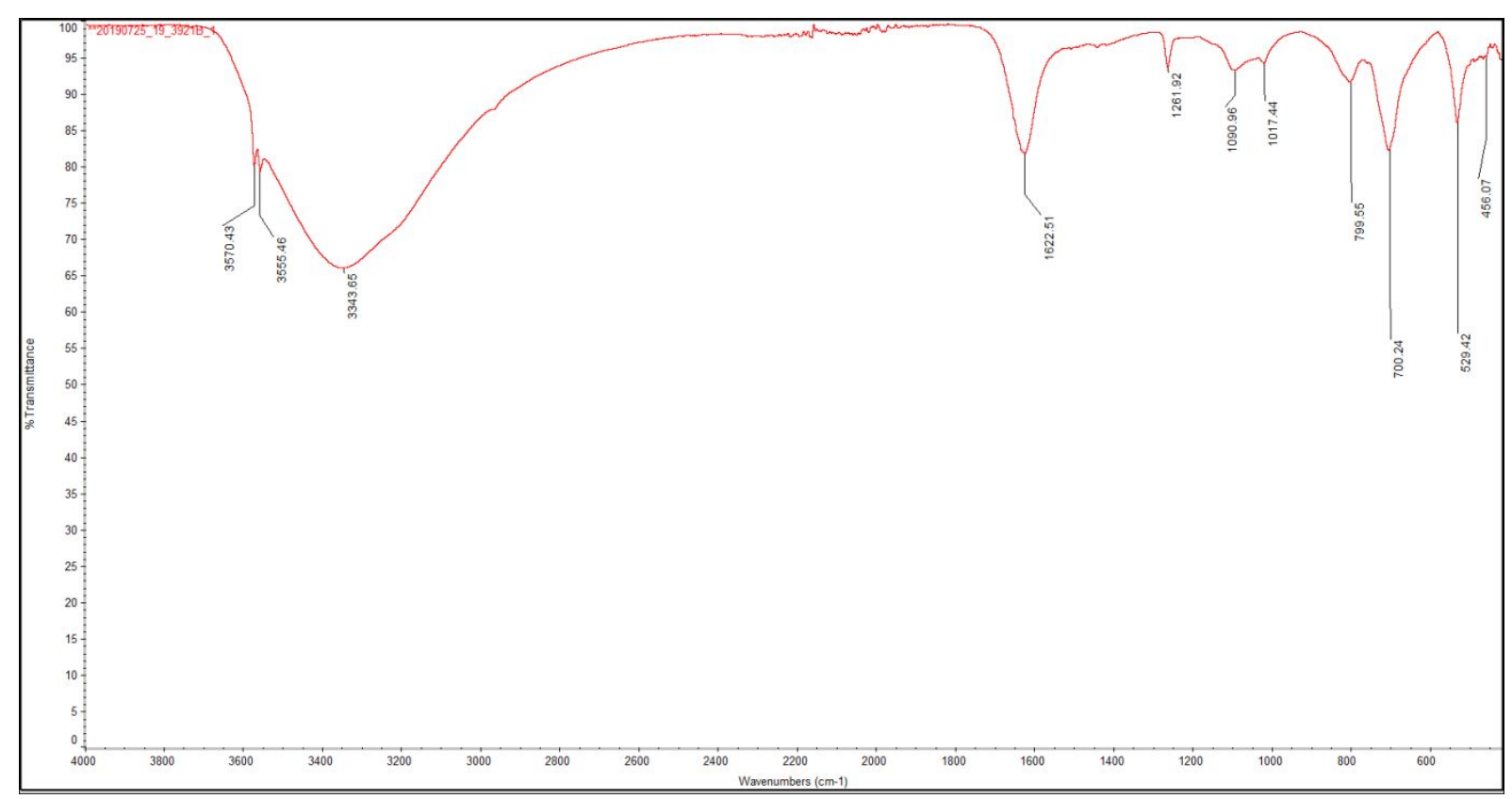

Figure S3. FTIR-ATR spectra of 3 .

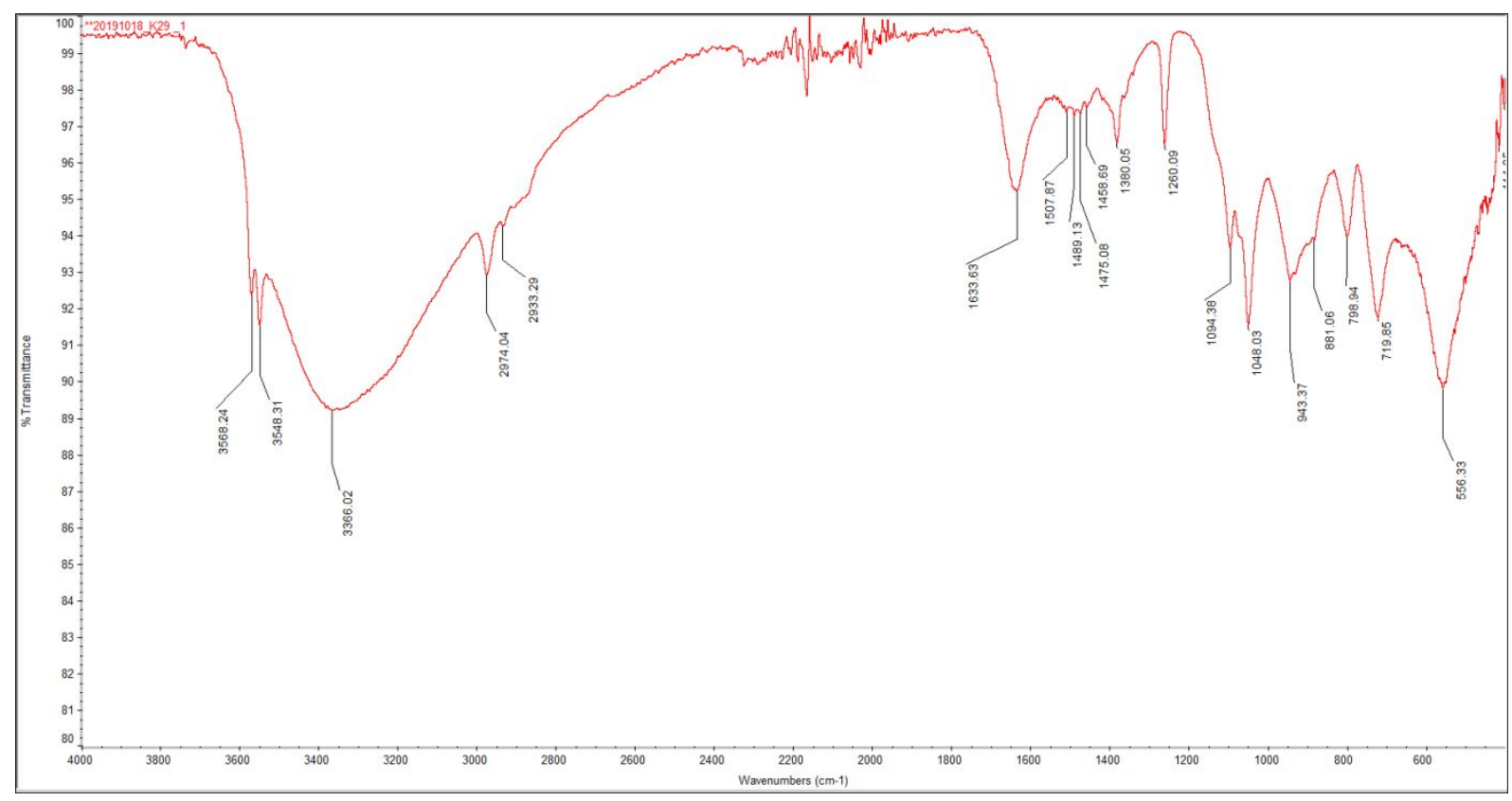

Figure S4. FTIR-ATR spectra of 4. 


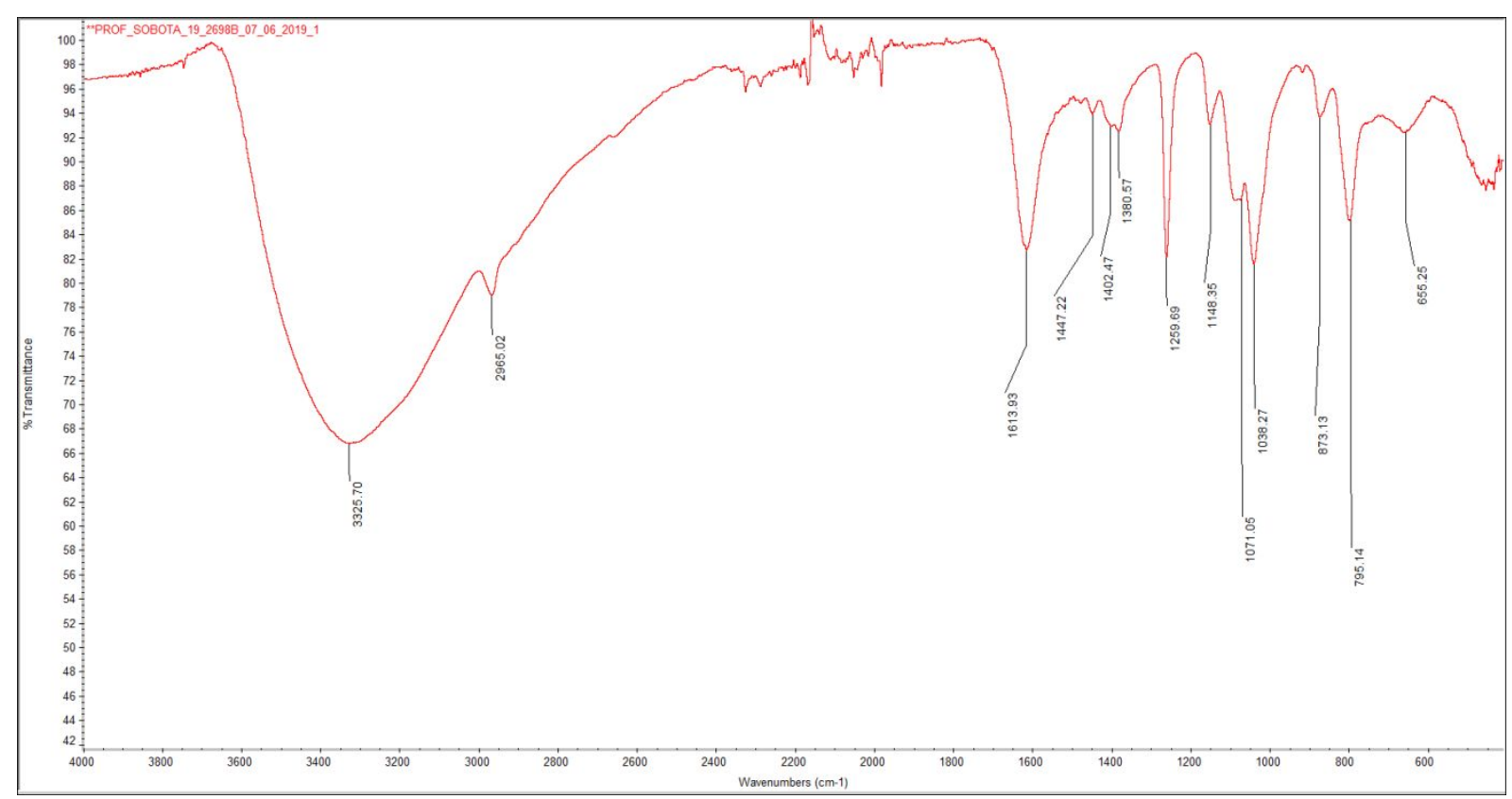

Figure S5. FTIR-ATR spectra of 5

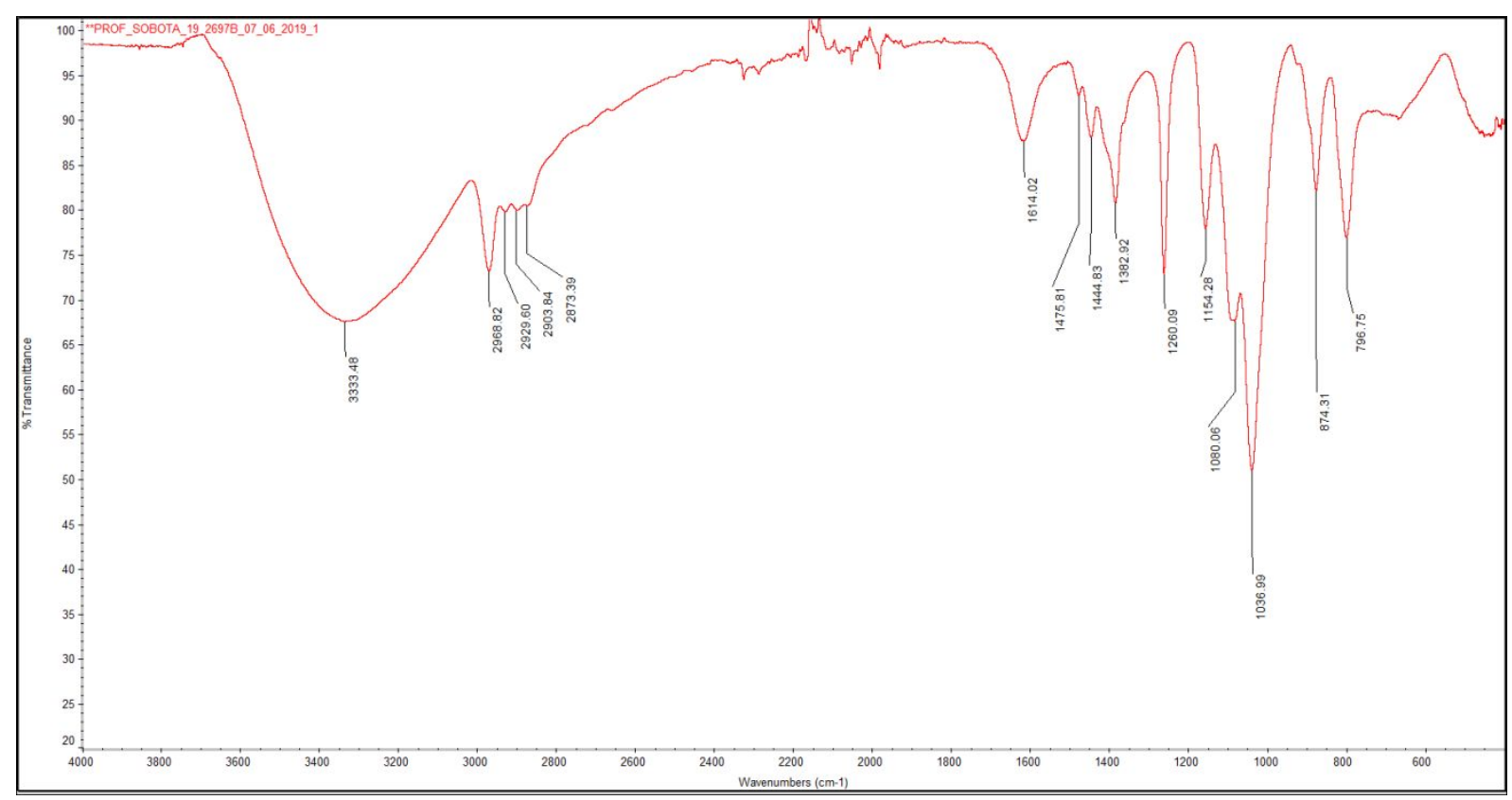

Figure S6. FTIR-ATR spectra of 6. 

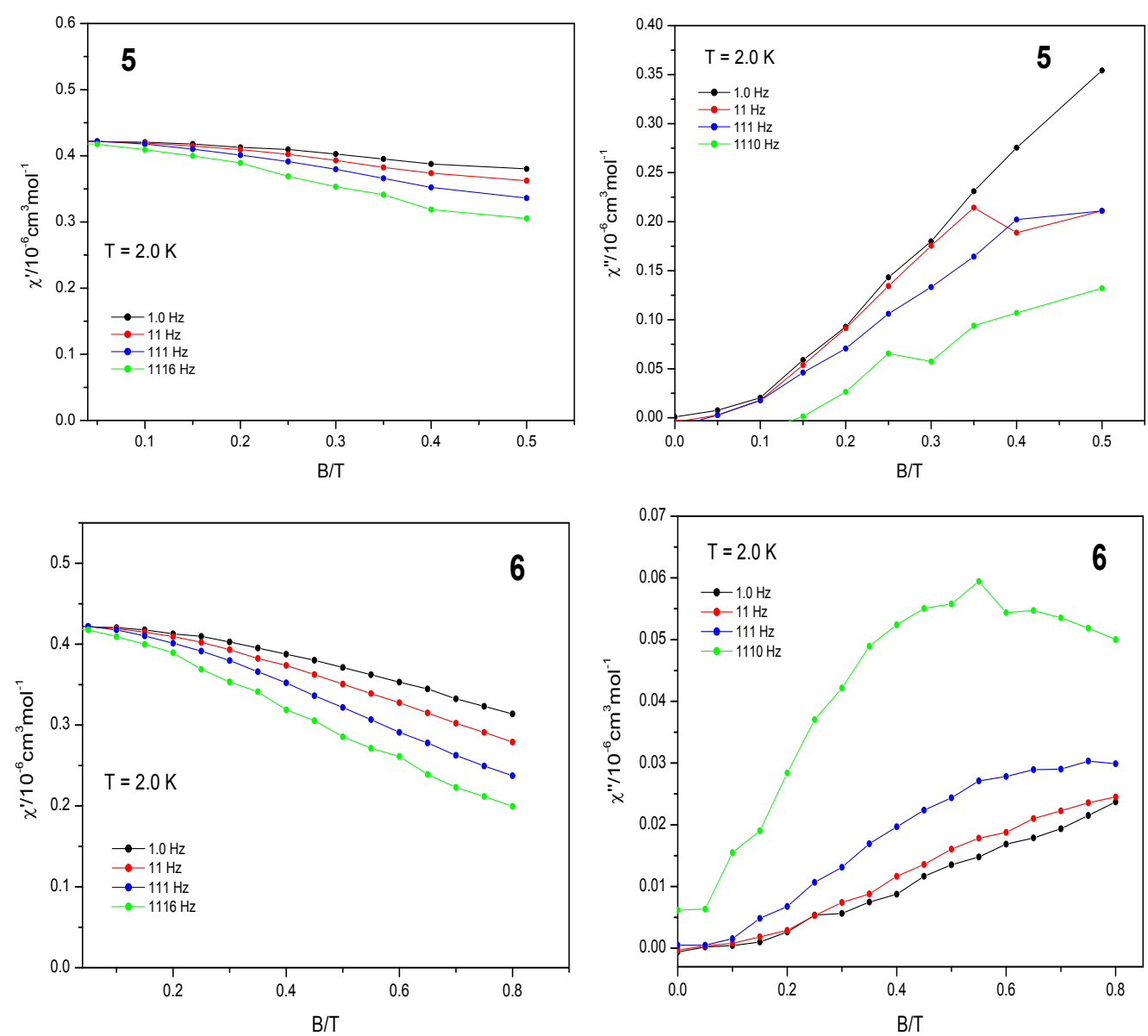

Figure S7. Field dependence of AC susceptibility components for 5 and 6 at $T=2.0 \mathrm{~K}$ for a set of frequencies of AC field. Lines serve as a visual guide.
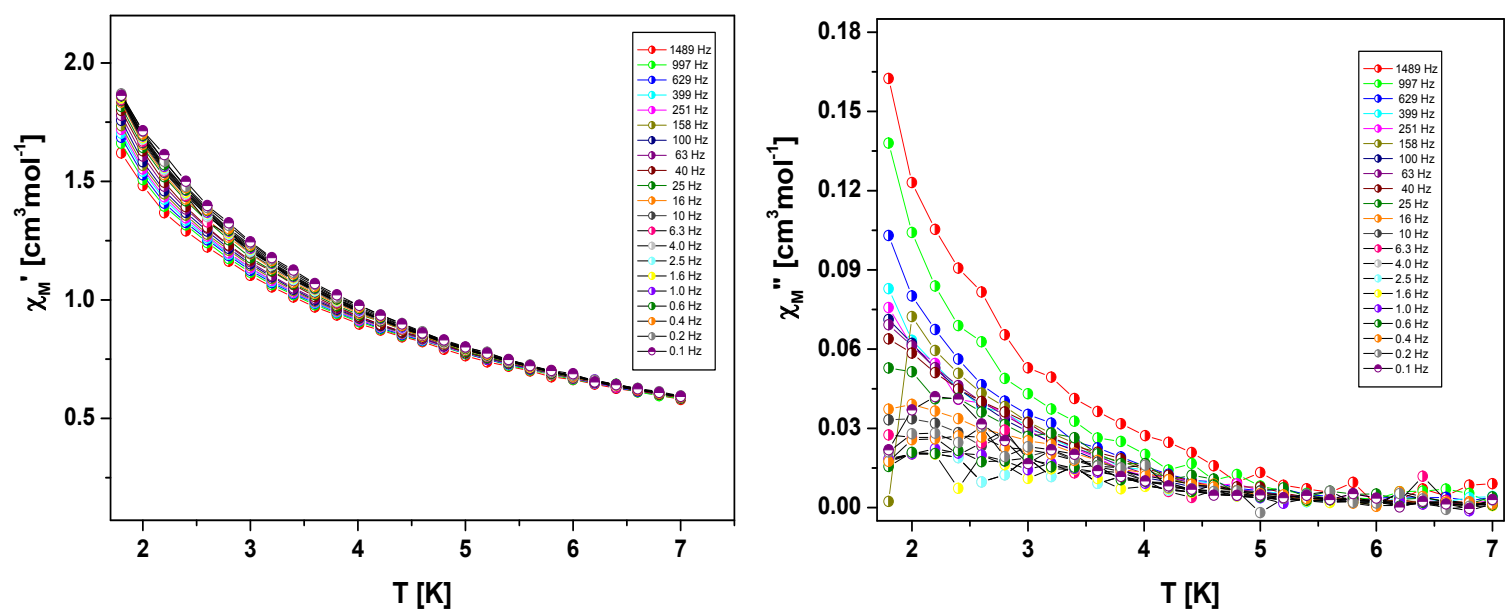

Figure S8. Temperature dependence of the AC susceptibility components for $\mathbf{6}$ for frequencies ranging between $0.1-1500 \mathrm{~Hz}$. 